
allemande

$52-2 \mid 2020$

Les traités de paix en Europe centrale : quels potentiels pour quelles réalisations?

\title{
Peter-Paul BÄNZIGER, Die Moderne als Erlebnis. Eine Geschichte der Konsum- und Arbeitsgesellschaft
} 1840-1940

Göttingen, Wallstein Verlag, 2020

Michel Hau

\section{(2) OpenEdition \\ Journals}

Édition électronique

URL : https://journals.openedition.org/allemagne/2491

DOI : 10.4000/allemagne.2491

ISSN : 2605-7913

\section{Éditeur}

Société d'études allemandes

Édition imprimée

Date de publication : 31 décembre 2020

Pagination : 444-445

ISSN : 0035-0974

Référence électronique

Michel Hau, «Peter-Paul Bänzıgen, Die Moderne als Erlebnis. Eine Geschichte der Konsum- und

Arbeitsgesellschaft 1840-1940 », Revue d'Allemagne et des pays de langue allemande [En ligne], 52-2 I

2020, mis en ligne le 31 décembre 2020, consulté le 20 février 2022. URL : http://

journals.openedition.org/allemagne/2491 ; DOI : https://doi.org/10.4000/allemagne.2491 
"renaître» à chaque série de représentations. Ce sera alors le passage officiellement assumé à l'ère de la mise en scène (fin du XIXe siècle) que Fatima Saadi présente et analyse dans son indispensable conclusion.

Lire cet ouvrage permet donc d'avoir un regard privilégié sur une phase décisive de l'évolution du théâtre européen, mais aussi sur la création théâtrale en général à travers la multiplicité de ses constituants textuels et scéniques. Enfin, et ce n'est pas le moins important, il nous permet de constater à quel point la création théâtrale et la réflexion qui l'accompagne sont un marqueur privilégié, voire même un acteur direct de l'évolution sociale et de l'échange interculturel. Le XVIIle siècle européen est à cet égard d'une richesse étonnante et il mérite de ce fait toute notre attention, en un temps où la peur de l'autre et le repli sur soi semblent de plus en plus motiver les esprits à l'échelle du monde. Toute notre reconnaissance donc à Fatima Saadi d'avoir porté ce regard à la fois aigu et passionné sur la création théâtrale européenne.

Jean-Jacques Alcandre

Peter-Paul BÄNZIGER, Die Moderne als Erlebnis. Eine Geschichte der Konsum- und Arbeitsgesellschaft 1840-1940, Göttingen, Wallstein Verlag, 2020, 452 p.

Peter-Paul Bänziger, qui enseigne actuellement en histoire contemporaine à l'Université de Bâle, nous livre dans cet ouvrage une histoire du vécu quotidien. Son enquête porte sur l'espace germanophone pendant la période de modernisation qui s'étend des révolutions de 1848 à la Seconde Guerre mondiale. Il a étudié pour cela une centaine de journaux intimes et de recueils de lettres conservés dans divers centres d'archives comme le Leo Baeck Institute de New York, le Deutsches Tagebuch Archiv d'Emmendingen, ou la collection Frauennachlässe de Vienne. Ces ego-documents, qui émanent surtout d'un milieu d'artisans et de bourgeois, retracent des destinées multiples. L'auteur les a fondues dans une sorte de biographie collective structurée selon un plan thématique, de façon à faire ressortir l'évolution d'une culture subjective commune. À travers des conditions de vie différentes, les contemporains partagent un même répertoire de représentations et de modes d'action.

L'exposé, très détaillé, transporte le lecteur dans un monde parfois éloigné du nôtre, tant pour les modes de vie que pour les façons de penser, mais sur une période suffisamment longue pour nous faire saisir toute l'évolution qui a abouti à notre présent. Sans chercher à appliquer les techniques statistiques à son corpus de données, l'auteur recourt à la méthode du commentaire littéraire. Son étude, solidement structurée et confrontée à une riche bibliographie, constitue une preuve, s'il en était besoin, de la valeur heuristique que possède une explication de texte riche de comparaisons et de nuances. Peter-Paul Bänziger nous fait pénétrer au plus intime de la vie personnelle de ses personnages, artisans, enfants d'industriels, pasteurs, employés, domestiques, etc. Les transformations de leur vécu sont décrites en considérant deux domaines essentiels, celui de la production et celui de la consommation.

La modernisation s'accélère durant les deux décennies de la fin du XIXe siècle. Les journaux personnels s'inscrivent moins que par le passé dans la perspective à long terme d'une biographie et davantage dans le vécu instantané (Erlebnis). Les éléments de permanence s'estompent. La maison familiale perd sa fonction ancienne de lieu de production et de consommation. Le logement se déconnecte du lieu de travail. L'ethos 
de l'activité laborieuse (Arbeitsamkeit) subsiste, mais, avec l'industrialisation, la fierté de l'artisan pour le bel objet est remplacée par le culte de la productivité et le souci de la compétitivité. Le salariat se développe et gagne le monde de la bourgeoisie.

La consommation cesse d'être simplement la contrepartie passive de la production: elle devient une forme d'activité destinée à équilibrer le travail, comme le préconisent les préceptes d'hygiène diffusés à la fin du XIXe siècle. Le congé d'été devient un élément important du calendrier chez les gens aisés. Si le luxe est toujours - au moins dans la rhétorique - condamné, les distractions ne sont nullement réprouvées, même quand elles se déroulent hors du cadre familial. Dans les journaux personnels, le théâtre, le sport, la mode, les voyages touristiques en Italie et le cinéma prennent une place croissante. La consommation est perçue de plus en plus comme une grandeur fondamentale dans les calculs des économistes. La question essentielle n'est plus de modérer les appétits selon la morale traditionnelle mais de faire en sorte que l'argent dépensé nourrisse l'économie nationale. Les sciences sociales introduisent le concept de niveau de vie.

L'auteur note que toutes ces évolutions ne débouchent pas encore pour autant sur l'individualisme postmoderne: si le modèle très contraignant de la famille souche a cédé la place à celui, plus libéral, de la famille nucléaire, cette dernière apparaît à l'ensemble des diaristes de la période comme le centre de la vie quotidienne et le but à atteindre. Parmi les critères de réussite s'ajoute même la transmission aux héritiers d'un patrimoine. Une comparaison avec les espaces situés hors du monde germanophone montrerait peut-être que cette orientation reste plus nette dans l'Europe rhénane que, par exemple, dans le monde anglo-saxon. Mais cela reste à vérifier à partir du même type de source et selon les mêmes méthodes.

Michel Hau

Bernd WEGNER, Das deutsche Paris. Der Blick der Besatzer 1940-1944, Paderborn, Schoeningh, 2019, 259 p.

Der Blick der Besatzer, ouvrage écrit par l'historien Bernd Wegner, richement illustré par des documents authentiques, frappe dès l'abord par son originalité. À commencer par celle de l'objet d'étude: l'occupation allemande de la capitale durant la Seconde Guerre mondiale a été certes souvent analysée du point de vue du Paris occupé, mais beaucoup moins de celui de l'occupant. Originalité de la démarche, ensuite: s'appuyant tout à la fois sur un nombre impressionnant de lettres, de témoignages individuels, de directives, d'affiches et de photographies publiques ou privées, Bernd Wegner parvient très concrètement à nous replonger dans l'ambiance si particulière et paradoxale des années d'occupation, mêlant une approche chronologique et thématique de son sujet. Le lecteur assiste, pas à pas, à l'évolution de la situation au fil des semaines, des mois et des années, ce qui n'empêche pas l'auteur d'établir de constants parallèles (on serait tenté de dire des va-et-vient) entre les différentes phases de l'occupation allemande.

Ces périodes successives s'éclairent ainsi mutuellement: l'attentisme français de la période qui précéda immédiatement l'arrivée des armées allemandes; la désertion, dans un premier temps, par les Parisiens aisés; puis l'arrivée des troupes allemandes et un certain soulagement, de part et d'autre, en constatant qu'un redémarrage de la vie économique et sociale était possible; dès lors une forme de sidération allemande 